\title{
What Are Predictors for Persistence in Childhood Stuttering?
}

\author{
Bridget Walsh, Ph.D., CCC-SLP, ${ }^{1}$ Evan Usler, Ph.D., ${ }^{2}$ Anna Bostian, M.S., ${ }^{3}$ \\ Ranjini Mohan, Ph.D., CCC-SLP, ${ }^{4}$ Katelyn Lippitt Gerwin, M.S., CCC-SLP, ${ }^{3}$ \\ Barbara Brown, M.S., CCC-SLP, 3 Christine Weber, Ph.D., CCC-SLP, 3 and \\ Anne Smith, Ph.D. ${ }^{3}$
}

Learning Outcomes: As a result of this activity, the reader will be able to (1) summarize the general framework behind the multifactorial dynamic pathways (MDP) theory of stuttering; (2) discuss predictive factors for stuttering persistence from behavioral and physiological studies of speech motor control, linguistic, and emotional processing in preschool children who stutter; (3) list several clinical assessments that could be

${ }^{1}$ Department of Communicative Sciences and Disorders, Michigan State University, East Lansing, Michigan; ${ }^{2}$ Department of Speech, Language, and Hearing Sciences, Speech and Feeding Disorders Laboratory, Boston University, MGH Institute of Health Professions, Boston, Massachusetts; ${ }^{3}$ Department of Speech, Language, and Hearing Sciences, Purdue University, West Lafayette, Indiana; ${ }^{4}$ Department of Communication Disorders, Texas State University, San Marcos, Texas.

Address for correspondence: Bridget Walsh, Ph.D., CCC-SLP, Department of Communicative Sciences and Disorders, Michigan State University, 1026 Red Cedar
Road, Oyer Speech and Hearing Building, East Lansing, MI 48824 (e-mail: walshb16@msu.edu).

Stuttering through the Lifespan: Questions and Answers; Guest Editor, Vivian Sisskin, MS, CCC-SLP, BCS-F.

Semin Speech Lang 2018;39:299-312. Copyright (C) 2018 by Thieme Medical Publishers, Inc., 333 Seventh Avenue, New York, NY 10001, USA. Tel: +1(212) 5844662.

DOI: https://doi.org/10.1055/s-0038-1667159.

ISSN 0734-0478. 
incorporated into a diagnostic battery to help predict whether a child is at a greater risk for stuttering persistence.

Our work to determine which factors can be used to determine the likelihood that stuttering will persist in preschool children is framed within the multifactorial dynamic pathways (MDP) theory, our attempt to integrate current knowledge about how stuttering develops. ${ }^{1}$ At the core of our theoretical account is the assertion that stuttering develops essentially as an impairment in speech sensorimotor processes that is strongly influenced by dynamic interactions among motor, language, and emotional domains. Another critical aspect of MDP theory is a strong emphasis on the complex maturational processes ongoing during the period in childhood in which stuttering initially appears and then takes its course either to recovery or persistence (see Fig. 2 in Smith and Weber). ${ }^{1} \mathrm{We}$ emphasize that children who begin to stutter follow heterogeneous "pathways" through this developmental period, during which different factors influence the child's eventual stuttering status.

Our research team employs this theoretical framework to guide both experimental and clinical approaches to the problem of developing a predictive battery of tests to use in young children who are stuttering (CWS). Fig. 1 summarizes the theoretical, experimental, and clinical levels of inquiry in our research on early stuttering. This research program, which we have called the Purdue Stuttering Project, has been funded by the National Institutes of Health, Institute on Deafness and Other Communicative Disorders since the late 1980s. We initially investigated the physiological correlates of stuttering in adults. Over the years, we learned, from the work of our laboratory and many others, how stuttering is expressed in central neurophysiological, peripheral physiological, and behavioral levels in adults. A key conclusion drawn from the work in adults who stutter is that stuttering is not "caused" by a single factor, such as atypical development of a specific brain region or heightened muscle activation during speech. Instead the peripheral and central physiological correlates of stuttering in adults are complex and widespread among many different functional systems.

In the last two 5-year research plans of the Purdue Stuttering Project, we designed and implemented longitudinal studies of preschool CWS and age-matched, nonstuttering peers. The two 5-year projects differed in a few significant ways; thus, we will refer to them as Phases I and II. In both phases combined, we recruited and followed cohorts of approximately 150 CWS and approximately 70 children who do not stutter (CWNS) in yearly visits to our laboratories. In Phase I, subsets of CWS and CWNS were recruited and followed longitudinally at the University of Iowa with Dr. Tricia Zebrowski as coinvestigator. The children participated in a wide range of evaluations, including careful assessment of their stuttering behaviors, linguistic skills, and a variety of other clinical measures. They also participated in experiments to probe development in the motor and language domains. We assessed neural processing during language perception tasks. In other experiments, we recorded muscle activity and articulatory movements during speech and other motor tasks. In longitudinal Phase II, we introduced an experimental arm to investigate emotional factors in early stuttering using behavioral and physiological (recording autonomic nervous system activity) measures during speech and other behaviors. In this article, we present a summary of our current, multileveled findings concerning factors related to persistence and recovery of stuttering in childhood. The organization of this study follows the diagram in Fig. 1 in discussing our approach and findings at theoretical, experimental, and clinical levels, and considers predictive factors within the motor, linguistic, and emotional domains.

\section{OVERALL GOALS, STUDY DESIGN, AND STUTTERING DIAGNOSIS}

A key element of the Purdue Stuttering Project Phases I and II is its prospective cohort design. 


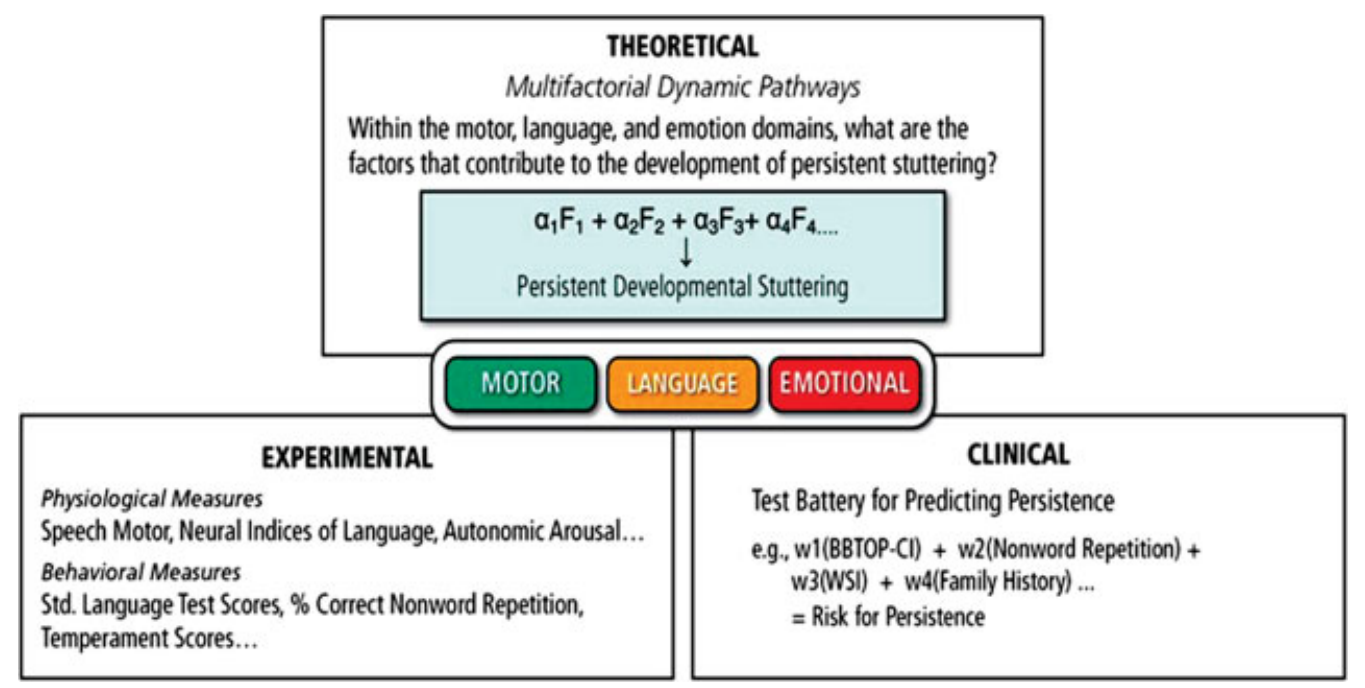

Figure 1 Our multilayered research framework. At a theoretical level, we wish to understand the factors in the motor, language, and emotional domains that contribute to the onset and persistence of stuttering. At the experimental level, our strategy combines behavioral/clinical and physiological measures to reveal significant factors associated with stuttering persistence or recovery. Finally, a future translational goal is to develop a predictive model that incorporates clinical and physiological variables to estimate risk for stuttering persistence.

Stuttering and nonstuttering children typically were recruited into our project when they were 4 to 5 years old. They were then followed longitudinally in yearly visits until age 9 or 10 years. Critically, this design allowed us to obtain a final determination of their status, stuttering eventually persisted (CWS-eP) or recovered (CWS-eR). When eventual stuttering status was known for the older children, we could then "go back" to examine the results from clinical, behavioral, and physiological assessments in the first year when they entered the project. Using this method, we could determine if, for example, CWS at age 4 to 5 years who had low scores on speech motor coordination, or phonological abilities, or nonword repetition tasks, were more likely to persist in stuttering.

Clearly a fundamentally important issue is the determination of whether a child is stuttering or not at each yearly visit. The lead speechlanguage pathologist (SLP) for the project assessed the children who were thought to stutter on entry to the project. During the initial visits with each child, we determined whether they met our diagnostic criteria for stuttering and, if so, could be enrolled in the longitudinal study. We used Yairi and Ambrose's three criteria for inclusion in the CWS cohort. ${ }^{2}$ In our experience, several factors were critical in determining the child's initial stuttering status, as well as the child's status in each subsequent year of participation in the study (i.e., recovered or persisting).

Obtaining a representative speech sample was an essential element in the diagnostic process and required collaboration with caregivers to ensure accurate assessment of the frequency and type of stuttering-like disfluencies (SLDs) in the child's speech. We obtained two speech samples from each child, the first in a play session with a parent or caregiver and the second with a clinician. This allowed us to analyze disfluencies in more than one speaking context. As recommended by Yairi and Ambrose, we obtained samples of 750 to 1,000 words over these two contexts. ${ }^{3}$ Clinicians may find that a shorter sample (i.e., $\sim 600$ syllables) is sufficient to gain an accurate picture of stuttering severity for many CWS; however, for children whose stuttering may be mild, or demonstrate avoidance of certain words, a longer sample is likely to yield a more accurate diagnosis. An established metric that we use to 
help diagnose stuttering in children is 3 or more SLDs per 100 syllables of conversational speech. From these speech samples, we also computed a weighted index of SLDs (WSI) for each child. ${ }^{4}$ This measure is particularly sensitive, because it captures not only the frequency of SLDs but also the number of iterations or repetitions and the specific type of SLD (e.g., dysrhythmic phonations such as blocks or prolongations, part and whole single-syllable word repetitions). The WSI assigns a higher weight to dysrhythmic phonations, given that these particular SLDs are absent or rare in the speech of typically fluent children. A WSI of 4.0 or higher indicates stuttering.

We obtained stuttering severity ratings on an 8 -point scale $(0-1=$ normal; $2-3=$ mild stuttering; $4-5=$ moderate stuttering; $6-7=$ severe stuttering) from caregivers and the project SLP during each visit. In a few instances in the speech sample analyses, we obtained less than 3 SLDs/100 syllables of speech, although both the caregiver and clinician rated the child a 2 (mild) or higher in severity. Additional information was obtained in these cases. The child was observed in other speaking contexts, for example, during conversations with siblings or while participating in experimental tasks as part of the study. The lead SLP used these observations, together with caregiver information provided during interviews and results from the Test of Childhood Stuttering ${ }^{5}$ (TOCS, see later) to diagnose a child as a CWS (or a CWNS) and, in later years, his/her status as persisting or recovered.

Speech samples are invaluable for obtaining comprehensive fluency profiles and expressive language abilities, yet they are admittedly time consuming. In Phase II, we added the TOCS, a norm-referenced stuttering assessment, to our diagnostic battery. The speech fluency component of this measure assesses fluency in structured contexts, for example, at the word, sentence, or conversational levels. Lower TOCS scores indicate more severe stuttering. Observational rating scales completed by caregivers assess a child's fluency and related behaviors in different environments. Such information is important to assess the child's willingness to speak in situations such as a preschool classroom or at family gatherings and to determine if anxiety or avoidance behaviors related to stuttering are present.

We recently compared the number of children diagnosed as stuttering both by the project SLP and caregiver during their initial visits of Phase II (i.e., child received a 2 or higher on the severity scale) with the results from the TOCS and WSI. Among the 51 children with final diagnoses of stuttering by the project SLP, the WSI identified approximately $86 \%$ or 44 children as stuttering and the TOCS identified over $90 \%$, or 46 children as stuttering. Two children presenting with mild stuttering were not identified with either of these measures.

We computed Pearson's correlation coefficients to examine the relationships among WSI and TOCS scores and parent and clinician ratings from this cohort of $51 \mathrm{CWS}$. The results are provided in Table 1 . Scores from the TOCS and WSI were moderately negatively correlated; higher WSIs and lower TOCS scores indicate more severe stuttering, while stronger correlations were observed between these measures and clinician ratings. We did not find a significant relationship between parent ratings and either the TOCS or WSI, although parent and clinician ratings were modestly correlated. These findings suggest that the TOCS and WSI tap into some overlapping and some independent phenomena related to the child's stuttering behaviors and experience. The higher correlation $(r=0.66)$ between the WSI and clinician rating also suggests that the clinician's ratings are more closely tied to the type and frequency of SLDs compared with the parent ratings $(r=0.24)$. In our experience, caregivers' qualitative input regarding their child's

\begin{tabular}{|c|c|c|c|c|}
\hline Measure & TOCS & WSI & $\begin{array}{l}\text { Parent } \\
\text { rating }\end{array}$ & $\begin{array}{l}\text { Clinician } \\
\text { rating }\end{array}$ \\
\hline TOCS & - & & & \\
\hline WSI & $-0.45^{\mathrm{a}}$ & - & & \\
\hline $\begin{array}{l}\text { Parent } \\
\text { rating }\end{array}$ & -0.12 & 0.24 & - & \\
\hline $\begin{array}{l}\text { Clinician } \\
\text { Rating }\end{array}$ & $-0.57^{a}$ & $0.66^{a}$ & $0.39^{a}$ & - \\
\hline
\end{tabular}

Abbreviations: TOCS, Test of Childhood Stuttering; WSI, weighted index of stuttering-like dysfluencies.

${ }^{a}$ Correlation is significant at the 0.01 level (two-tailed). 
fluency fluctuations under different demands and communicative contexts, along with details regarding a child's reactions to stuttering, are essential components of the diagnosis. These findings also highlight the importance of clinical experience and intuition as integral pieces of evidence-based stuttering diagnostics. The two children diagnosed by the project SLP with mild stuttering, but not by the WSI or TOCS, have not met criteria for recovery, so are considered to be persisting.

\section{EXPERIMENTAL LEVEL: NEUROBIOLOGICAL CLUES INTO THE PROCESSES OF RECOVERY AND PERSISTENCE}

By discovering the peripheral and central physiological markers of the emergence of persistent stuttering in childhood (and characteristics of the pathway to recovery), we unearth potentially important clues as to what measures might be the most promising predictors of stuttering chronicity. These predictors are important from a theoretical standpoint as they advance our understanding of stuttering, and they may also have important clinical implications for improving diagnostic and treatment protocols. We readily acknowledge that the experimental methods used in our physiological investigations of stuttering are not available to most clinicians. Yet the past decade has seen a dramatic increase in wearable biosensor technology. ${ }^{6}$ Several of the physiological signals that we collect in our laboratories (e.g., skin conductance, heart rate, and articulatory movement data; see the following sections) can be collected with disposable biosensors that transmit signals to devices such as a personal digital assistant or smart phone for analysis. Thus, empirical findings such as ours may guide the development of such devices for future clinical use and will have important implications for predicting the likelihood of stuttering persistence.

\section{ERP Correlates of Language Processing Associated with Recovery and Persistence}

Event-related brain potentials (ERPs) are electroencephalographic (EEG) signals that are averaged to produce a brain wave response pattern time-locked to the onset of a stimulus. Depending on the nature of the experimental task, ERPs are associated with sensory, linguistic, and cognitive processes. ${ }^{7}$ ERPs related to linguistic and emotional processing have been shown to be dynamic markers of development during the preschool and school-age yearsthe sensitive period of development in which spontaneous recovery from stuttering will or will not occur.

Earlier ERP studies from our group revealed atypical language processing in some adults who stutter compared with fluent adults (reviewed in Smith and Weber ${ }^{1}$ ). These findings suggested that, in the absence of overt language deficits, subclinical differences in neural activity for perceptual language processing may represent potential risk factors for persistent stuttering. ${ }^{1}$ We explored this through several prospective studies comparing ERP amplitude, latency, and distribution associated with semantic, syntactic, and phonological processing in CWS.

Kreidler and colleagues ${ }^{8}$ used ERPs as an index of semantic processing in 5-year-old CWS-eP, CWS-eR, and CWNS while they listened to sentences presented in a story context. Most of the CWS-eR exhibited a more mature pattern of ERP responses, similar to those seen in adult listeners, compared with CWS-eP. This finding suggested that future recovery from stuttering is associated with earlier maturation of semantic processes in the preschool years. In a study of 6- to 7year-old children, ERPs associated with syntactic processing were obtained from children already recovered from stuttering (CWS-Rec), children who were persisting (CWS-Per), and fluent controls. ${ }^{9}$ We measured ERPs elicited by syntactic (phrase structure) violations within Jabberwocky sentences. Jabberwocky sentences consist of English function words but nonsense content words. This paradigm was used to isolate syntactic processing by removing semantic information. We found the expected pattern of ERP responses to syntactic violations in most of the CWS-Rec and CWNS; however, we observed an atypical ERP response pattern in some of the CWS-Per. This finding suggests that neural mechanisms associated with 
syntactic processing are less mature in schoolage children with persistent stuttering.

Finally, in a study of phonological processing in 7- to 8-year-old CWS-Per, CWS-Rec, and CWNS, we examined ERPs elicited to pairs of rhyming and nonrhyming nonwords presented through speakers. ${ }^{10}$ Nonwords were selected to index phonological processing in the absence of semantic information. When the pairs of nonwords rhymed, the recovered and control children displayed the expected neural indices of early rhyme segmentation and rehearsal over anterior brain areas (called the anterior onset rhyme effect), but this effect was absent in the persisting children. This finding suggests atypical neural patterns underlying phonological processing in some CWS-Per. Furthermore, fluent controls exhibited the anterior-onset rhyme effect over both hemispheres, but this effect was only observed over the right hemisphere in the recovered children. Compared with typically developing children, hemispheric differences in ERP correlates of phonological processing were evident even after recovery from stuttering.

In summary, the use of ERPs elicited during receptive language tasks revealed that persistent childhood stuttering, even as early as the preschool years, is associated with atypical brain responses in terms of amplitude, latency, and distribution. These findings clearly point to a lag in the neurodevelopment of language for a significant proportion of CWS, with the path to recovery marked by a maturational shift toward more typical, adult-like ERP patterns of linguistic processing. Conversely, the persistence of stuttering is marked in some children by the continued presence of less mature, atypical patterns of brain activity.

\section{Neural Activity during Speech Production in Stuttering}

There is emerging evidence of atypical neuroanatomical development in CWS; however, we know little about brain activation during speech production. Functional near-infrared spectroscopy (fNIRS) is a noninvasive, child-friendly optical neuroimaging method that measures the relative concentration changes in oxygenated hemoglobin $(\mathrm{Oxy}-\mathrm{Hb})$ and deoxygenated
(Deoxy-Hb) hemoglobin in cerebral capillary blood. These hemodynamic activation patterns provide an indirect measure of cortical activity in the region and are not as susceptible to movement artifact as EEG recordings. Recently, Walsh and colleagues ${ }^{11}$ assessed hemodynamic patterns during overt speech production in school-age CWS-Per and CWNS with fNIRS. The children completed a picture description task while we recorded hemodynamic activity over neural regions integral to speech production and implicated in stuttering: inferior frontal gyrus (IFG), premotor cortex (PMC), and superior temporal gyrus.

A major finding from this cross-sectional study was the markedly different speech-related hemodynamic activation patterns between the two groups of children during fluent speech production. Although fluent controls showed clear speech-related activation of left dorsal IFG and left PMC, most CWS demonstrated the reverse, a deactivation over these left hemisphere regions. Earlier studies suggest that regions such as the left IFG comprising Broca's area develop atypically in some children who stutter. ${ }^{12,13}$ Our study is the first to demonstrate speech-related functional differences in brain activation patterns between groups of children who do and do not stutter.

An interesting question we wished to explore was whether or not the differences in brain activation patterns during speech could differentiate individual CWS and CWNS. Therefore, in a follow-up study, we explored whether discriminating features extracted from children's hemodynamic responses could be used to statistically classify individual CWSPer or CWNS into their respective speaker groups. ${ }^{14}$ Using a feature set extracted from $\mathrm{Oxy}-\mathrm{Hb}$ and Deoxy-Hb speech activation patterns from mostly left hemisphere fNIRS channels, we identified neurophysiological biomarkers of stuttering that distinguished CWS from CWNS with nearly 88\% accuracy. As a next step, we used the same classification algorithms on a new dataset from a group of CWS-Rec to determine whether their hemodynamic patterns would classify them as CWSPer or CWNS. Interestingly, the majority, $71 \%$, of these children classified as CWNS. 
Given that these children are no longer stuttering, we assume that normal speech function has returned. Our neurophysiological findings support this, as speech-related hemodynamic activity patterns from recovered children were, on average, more similar to children who had never stuttered than children who persisted. Yet, without longitudinal data, we can only speculate about a potential relationship between neurophysiology and stuttering behaviors.

\section{Physiological Studies in the Emotional Domain}

An increasing number of studies have been conducted in recent years using behavioral observation, caretaker reporting, and psychophysiological measures to investigate potential relationships between early childhood stuttering and emotional reactivity and regulation. ${ }^{15,16}$ Emotional reactivity refers to the arousal of cognitive, autonomic, and endocrine processes in response to a stimulus. This reaction, in turn, can be modulated by self-regulation. ${ }^{17}$ As part of the initial assessment battery, we obtain a temperament profile for each child using the short form of the Children's Behavior Questionnaire (CBQ). ${ }^{18}$ The CBQ is an established measure of temperament based on parental report to assess temperament characteristics. We also administered the KiddyCAT to each participant. ${ }^{19}$ This measure assesses a child's attitude about his or her communication through 12 yes/no questions. Children who receive higher scores on this measure are considered to have more negative attitudes about their speaking abilities.

Given the complexity of the potential relationship among emotional factors and stuttering, investigations integrating both behavioral and physiological measures may provide more fruitful insights. Recently, we and others have incorporated psychophysiological measures (e.g., electrophysiology, electrodermal, and heart rate measures) to observe and measure covert arousal responses from CWS during different communicative contexts. ${ }^{20-23}$ This approach permits us to examine important questions about whether some CWS show higher sympathetic arousal, particularly during speaking tasks, compared with CWNS, and if
CWS show higher sympathetic arousal during moments of stuttering compared with when they are speaking fluently.

To date, we have recorded skin conductance and blood pulse volume measures, sensitive indices of sympathetic nervous system arousal, in over 70 preschoolers, 46 CWS, and $25 \mathrm{CWNS}$. We found that CWS had significantly higher skin conductance levels compared with their fluent peers during both speech and nonspeech (i.e., blowing on a party favor and jaw wagging) experimental tasks. ${ }^{22}$ The participants had similar skin conductance levels at resting baseline at the beginning of the experiment, which provides evidence that CWS do not have inherently higher arousal levels, rather the higher arousal levels appear to be task related. It is important to note that, although we find significant statistical differences between groups of CWS and CWNS in skin conductance levels, measures from the two groups are overlapping. A recent longitudinal study revealed significantly higher sympathetic arousal in CWS-eP compared with CWS-eR during a stressful naming task. ${ }^{24}$ More longitudinal research examining whether heightened sympathetic activity in some CWS represents an early biomarker of chronicity is merited.

In a follow-up study, we analyzed sympathetic arousal in a group of CWS who produced fluent and disfluent utterances during a picture description task. ${ }^{25}$ We noted larger skin conductance responses occurring at a greater frequency along with reduction in blood pulse volume amplitude (indicative of increased sympathetic arousal) during stuttering. Because stuttering awareness is emerging in these young children, ${ }^{26}$ we do not suggest higher arousal reflects anticipation of or undue anxiety about stuttering. Rather we propose that increased sympathetic arousal levels during speaking may contribute to instability in the speech systems of CWS, thus increasing the probability of breakdowns. On the other hand, phasic increases in arousal during stuttering moments may indicate awareness, at some level, of these involuntary disruptions. As suggested previously, through longitudinal investigations, we can determine whether heightened sympathetic arousal, particularly during disfluent speech production, is associated with chronic stuttering and a factor 
in the development of communication anxiety in those who stutter. ${ }^{27}$

In a separate study of emotional reactivity and regulation in 5- to 8-year-old CWS and $\mathrm{CWNS}^{28}$ we recorded ERPs elicited by images of children's faces that displayed negative emotions such as anger or fear to explore neural reactivity to aversive stimuli. In another condition, these threatening facial expressions were preceded by a mollifying auditory cue (e.g., the child heard "Joe is pretending" prior to seeing an angry face). This experimental design allowed us to measure the brain's initial response to the threatening stimuli (emotional reactivity), as well as any reduction of this ERP response by receiving information that neutralized the threat (emotional regulation). We examined a late positive ERP component ( 300-1,000 ms after face presentation) which has been correlated with emotional arousal. We found that the two groups of children reacted to the aversive stimuli similarly, exhibiting a higher amplitude ERP response to threatening faces compared with neutral faces. The amplitude of this ERP was downregulated when the threatening face was preceded by a neutralizing audio cue in both CWS and CWNS. We note that our findings are not mutually exclusive from behavioral observation and parental reporting of subtle differences in emotional processes between young CWS and CWNS. ${ }^{29,30}$

\section{Physiological Studies in the Motor Domain}

Early studies in adults who stutter revealed differences in the speed, timing, and coordination of articulatory movements during perceptually fluent speech. ${ }^{31}$ At that time, it was unclear, however, whether the differences in speech motor parameters observed in adults who stutter were present near the onset of stuttering, distinguishing CWS from fluent peers. In our longitudinal work with preschool aged CWS, we have recorded articulatory movements of the jaw and lips with small infrared markers during speech production. We assess fundamental characteristics of speech movements, including duration, amplitude, velocity, and a measure that captures the con- sistency of coordination across repeated productions, the lip aperture (LA) index. ${ }^{32-34}$

During these experiments, children listen to, then produce nonwords and sentences varying in length and linguistic complexity to assess the potential effects of phonological or syntactic complexity on speech movement and coordination. A seminal finding was that, on average, CWS as young as 4 to 5 years have less mature speech coordination compared with fluent peers even while speaking fluently. Increasing the length or complexity of the nonwords and sentences also resulted in lower performance accuracy in the group of CWS. The more complex stimuli proved challenging for many CWS, resulting in a significantly greater number of errors and disfluencies. Because we were not able to obtain full datasets from many CWS, we completed a follow-up study assessing speech motor performance during the production of simple structured sentences that most children could easily produce, for example, "Buy Bobby a puppy." ${ }^{34} \mathrm{We}$ obtained data from over 100 preschool children, including $58 \mathrm{CWS}$, allowing us to examine sex differences within each group. We found that boys who stutter produced speech with significantly reduced movement amplitudes and velocities and less-coordinated articulatory movement patterns. Girls who stutter did not differ from their fluent peers on these speech motor measures. Thus at age 4 to 5 years, boys but not girls who stutter are significantly lagging their normally fluent peers in speech motor maturation. We speculate that functional differences in speech motor control processes may be a key factor in the well-documented, higher probability of persistence in stuttering in males.

Again, however, these cross-sectional studies of groups of CWS and CWNS suggest potentially significant factors for persistence that must be examined in relation to eventual persistence or recovery. Therefore, these novel findings inspired the important question of the trajectories of speech motor development in CWS who will recover versus those who persist. In a recent cross-sectional study, ${ }^{35} 5$ - to 7 -yearold children were assigned to three groups: 21 CWS-Per, 15 CWS-Rec, and 31 CWNS. Participants produced four declarative sentences that systematically varied in length and 
syntactic complexity in response to an auditory model. CWS-Per exhibited significantly lower coordination consistency, quantified by the LA index, compared with CWS-Rec and CWNS, while similar coordination consistency was observed for these latter two groups. It appeared that the speech motor abilities of CWS-Rec had "caught up" to that of typically developing children after an initial lag in speech motor performance. ${ }^{32}$ We concluded that maturation of speech motor coordination to a degree similar to that of typical children appears to be a characteristic of recovery from stuttering by the age of 5 to 7 years, while CWS-Per continue to lag in speech motor development.

\section{EXPLORING THE PREDICTIVE POTENTIAL OF CLINICAL TOOLS}

As noted in the first section, our standard protocol included assessment of the children in each cohort with a battery of clinical tests when they entered the project. We examined whether scores CWS received on these tests on entry to the project at age 4 to 5 years were predictive of their eventual stuttering status. We noted earlier that ERP investigations of CWS revealed differences in brain activation patterns during the processing of syntactic, semantic, and phonological information. An obvious question is whether behavioral probes of expressive and receptive language, phonology, articulation, and verbal working memory abilities are also associated with recovery or persistence in preschool children who stutter. ${ }^{36}$ Sixty-five children ages 3, 9 to 5 , and 8 years participated in this longitudinal study: 40 CWS and 25 CWNS. In addition to our fluency diagnostic battery, Phase I participants completed the following assessments during their initial visit: the Test of Auditory Comprehension of Language, 3rd edition $^{37}$ (TACL-3) to measure receptive language, the Structured Photographic Expressive Language Test, 3rd Edition ${ }^{38}$ (SPELT-3) to measure expressive language, the BanksonBernthal Test of Phonology-Consonant Inventory subtest ${ }^{39}$ (BBTOP-CI) to assess articulation ability, the Nonword Repetition Test ${ }^{40}$ (NRT), an index of articulatory working memory and production of novel phonological sequences, and the Test of Auditory Perceptual
Skills-Revised auditory number memory and auditory word subtests ${ }^{41}$ (TAPS-R) to measure auditory working memory.

In subsequent years, 19 of the $40 \mathrm{CWS}$ were identified as children who eventually recovered and 21 as children who persisted in stuttering. We used scores from the initial assessment battery to explore potential differences in group means among CWNS, CWS-eP, and CWS-eR. We found no differences among CWS-eR and CWS-eP and CWNS on measures of receptive language, expressive language, and verbal working memory. It is important to note that although we observed subtle differences between CWS-eR and CWS-eP on ERP indices of language processing, these differences were not associated with clinical language impairments. We found that, as a group, CWS-eP scored significantly lower than CWNS and CWS-eR on the BBTOP-CI and the NRT task. A regression analysis revealed that scores on the BBTOP-CI and NRT significantly predicted future stuttering recovery or persistence of these preschool CWS. In line with earlier studies of phonological processing and stuttering, ${ }^{42}$ our findings suggest a role for phonological and articulatory skills along with planning and production of novel phonological sequences as potential predictive measures.

Another promising avenue for predicting the likelihood of persistence in stuttering using clinical assessments is the analysis of stuttering behaviors. Previous findings in 2- to 3-year-old CWS indicated that near onset, severity, and type of SLDs are similar for CWS-eP and CWS-eR. ${ }^{43}$ Obviously, with maturation CWS-eR and CWS-eP follow different trajectories in terms of the frequency and type of SLDs. Given that the majority of recovery takes place within 15 months after the average onset of 33 months, ${ }^{43}$ the 4 - to 5 -year period is a pivotal stage in terms of recovery and persistence. During the 4- to 5-year period, neurobehavioral systems are undergoing dramatic maturational changes, ${ }^{1}$ and clinicians employed in preschool and school settings must make consequential decisions on whether or not to treat a child displaying stuttering behaviors.

Bostian et $\mathrm{al}^{44}$ analyzed spontaneous speech samples collected during the initial visits 
from 74 to 4-year-old children recruited in Phase I. These samples were coded using the Systematic Analysis of Language Transcripts ${ }^{45}$ (SALT) for type of SLD (part- and singlesyllable word repetitions and dysrhythmic phonations) and other disfluencies (ODs, interjections, revisions, and multisyllabic word or phrase repetitions). We tabulated the frequency and maximum number of repetition units of SLDs and ODs and calculated a WSI for each child. In a prospective design, we examined group differences among 18 CWS-eP, 29 CWS-eR, and 27 CWNS on the measures collected during their first visit. As expected based on previous findings, the frequency and type of ODs did not distinguish the three groups of children. However, preliminary findings suggest that 4- to 5-year-old CWS-eP had significantly higher frequencies of SLDs, numbers of repetition units, and WSI scores compared with CWS-eR or control groups. These results suggest that there are significant differences in characteristics of SLDs displayed by groups of 4- to 5-year-old children who will eventually recover or persist. This is an important finding that validates the use of clinical measures of stuttering behaviors to identify 4to 5-year-old CWS at greater risk for stuttering persistence.

\section{CONCLUSION}

We have summarized major findings from our laboratory and others to explore current answers to the question of how we can predict the probability of persistence of early childhood stuttering. The issue is critical in the decision-making process of whether or not to treat a preschool child who is stuttering. The Purdue and the Illinois Stuttering Project ${ }^{3}$ are two relatively large-scale investigations in which preschool CWS have been followed longitudinally until final stuttering status was known. Results of studies from these two projects and other laboratories have added significant information about factors useful in predicting persistence. How do we synthesize results from these experimental and clinical assessments to guide the clinical decision-making process?

It is important to view new information within the context of existing and generally accepted risk factors for persistence: for example, family history of stuttering, later age of onset, longer time since onset, and sex-boys are more likely to persist. Our findings provide further evidence for the influence of sex on the incidence and persistence of stuttering. The average age at project entry for Phase I CWS was approximately 58 months (the average age of stuttering onset for these children was 36 months, so they had been stuttering, on average, for 22 months), with boys outnumbering girls by a ratio of nearly $3: 1,74 \%$ of the sample. The recovery rate for boys in this cohort was $42 \%$, while for girls it was significantly higher, 69\%. Results from physiological experiments have shed light on specific sex-linked differences in speech motor maturational trajectories. Boys aged 4 to 5 years lag girls in typical development of interarticulatory coordination, ${ }^{46}$ and some preschool boys who stutter produce speech with reduced articulatory coordination and with smaller amplitudes and lower velocities even when speaking fluently. ${ }^{34}$ Chang and her colleagues have reported differences in the relationship between white matter neural pathways and stuttering severity for boys and girls who stutter. ${ }^{13}$ Thus, we can state that sex is important in determining probability of persistence and is likely related to sex-linked differences in maturation of central speech motor control networks.

Another well-known predictor of persistence is a family history of stuttering. ${ }^{47}$ Consistent with earlier estimates, $53 \%$ of our Phase I cohort of children who persisted in stuttering reported having a first- or second-degree relative with persistent stuttering, while this percentage was much lower for children who recovered, $14 \%$. On the basis of their comprehensive review, Kraft and Yairi ${ }^{47}$ concluded that the genetic basis of familial transmission of stuttering is unknown. Despite the lack of knowledge of the specific genes that might be involved, a positive family history is another important variable to consider.

Turning to the three domains of inquiry, motor, linguistic, and emotion/temperament, which variables seem promising? In the motor domain, we have already alluded to developmental delays in speech sensorimotor systems, especially in boys who stutter. Nonword 
repetition performance, which has been widely used in assessing language status, also places demands on speech motor planning and execution processes. We presented evidence that nonword repetition performance is predictive of eventual stuttering status. ${ }^{36}$

In the linguistic domain, there is no single, consistent developmental profile of scores on standardized tests that is predictive of recovery/ persistence. We have reported evidence that lownormal scores on the BBTOP-CI have predictive power. ${ }^{36} \mathrm{ERP}$ work from our project, presented earlier, demonstrates that subtle differences in the functioning of the neural networks involved in processing linguistic information are related to eventual recovery or persistence. Thus, more targeted predictors of persistence related to language development are needed, and future behavioral and/or clinical investigations will explore the relationships among the markers of stuttering observed in brain activity during linguistic processing and behavioral performance during language production tasks.

Concerning the role of emotional factors in the development of persistent stuttering, recent studies in this domain using both electrophysiological and behavioral measures are promising. We demonstrated higher levels of sympathetic arousal in CWS in speech and nonspeech task performance. ${ }^{22}$ Physiological arousal is related to emotional and cognitive processes. In turn, increased arousal leads to increased instability in the speech motor system. Erdemir et $\mathrm{al}^{48}$ reported promising results showing that compared with CWNS and CWS-Rec, CWS-Per had slower articulation rates when generating a narrative describing a video clip eliciting negative emotion. They suggest that articulation rates following negative emotional stimuli in preschool CWS could be a factor that predicts persistence/recovery from stuttering. This finding deserves further investigation, because it explores the emotion/ motor link and employs a relatively simple behavioral assessment.

Results from longitudinal studies also point to careful analyses of the child's stuttering behaviors as an important predictive factor. Yairi and Ambrose ${ }^{3}$ reported that the frequency and type of SLDs near the onset of stuttering are not predictive of eventual persistence/reco- very. However, by the age of 4 to 5 years, the WSI score and the presence of certain types of SLDs are predictive of future status. ${ }^{44,49}$ Results from our laboratory ${ }^{44}$ suggest that a WSI $\geq 10$ at 4 to 5 years should be considered a risk indicator for persistence.

Our findings along with others support the practice of comprehensive assessments of CWS, a battery of tests that identifies relative strengths and weaknesses and the presence or absence of factors across domains that indicate risk for persistent stuttering. The evidence points to the need to take into account both the multifactorial and dynamic natures of stuttering development to determine which children are at greatest risk. We suggest that SLPs asses a constellation of factors for a given child to understand the unique profile he/she presents at age 4 to 5 years. The overwhelming evidence from our work and others ${ }^{3}$ is that persistent stuttering is not linked to a single profile of risk factors. For example, a child who persists may not necessarily have a known family history; therefore, other indicators, for example, delayed articulation abilities and/or attitudes toward speaking could provide a clinician with important clues about the potential for recovery. Our findings, taken together with earlier work, also emphasize the need to take into account the age of the child when assessing risk factors. For example, stuttering severity is not an indicator of persistence or recovery at age 2 to 3 years $^{50}$; however, it does provide important information for possible risk at age 4 to 5 years, ${ }^{44}$ when many children are initially evaluated for treatment.

Keeping in mind the heterogeneity of risk profiles as well as specific developmental milestones at age 4 to 5 years, a comprehensive assessment for determining risk of persistence will minimally include information about the child's sex, family history of persistent stuttering, language status (whether delayed or precocious $^{3}$ ), poor NRT performance, a WSI $\geq 10$, and/or evidence of negative attitudes toward his/her speech (e.g., assessed on the bases of parental report, TOCS, or KiddyCAT assessments). Unlike the equation shown in Fig. 1, this is a categorical list, for example, the answer is yes or no to each variable, and no weights are assigned to place greater or less 
emphasis on each item. An objective of our project is to generate a multivariate, weighted formula for assessing risk of persistence in preschoolers who stutter. The details of this effort, which involve the statistical method of structural equation modeling, are beyond the scope of this paper; however, we are working toward a translational goal of developing a prototypical predictive model to estimate risk for stuttering persistence using behavioral/clinical and physiological variables from our research studies. As we learn more, we hope that SLPs will have greater precision in predicting risk for stuttering persistence, so that priority for initiating fluency treatment can be made for those children most in need.

\section{ACKNOWLEDGMENTS}

This research was supported by the National Institute on Deafness and Other Communication Disorders (R01 DC00559 awarded to A.S. and C.W., and R03 DC013402 to B.W.). We wish to thank Janna Berlin, a research associate on the project, and Tricia Zebrowski and her colleagues at the University of Iowa for their collaboration on the Purdue Stuttering Project. Lastly, we are grateful to all of the children and their families whose participation made this research possible.

\section{DISCLOSURES}

B.W. is supported by NIH NIDCD grants R03 DC013402 and R01 DC00559. She has no relevant nonfinancial relationships to disclose. E.U. receives funding from an NIH NIDCD postdoctoral training grant T32 DC013017, PI: Christopher Moore. He has no relevant nonfinancial relationships to disclose.

A.B. has no relevant financial disclosures. She has no relevant nonfinancial relationships to disclose.

R.M. receives salary from Texas State University. She has no relevant nonfinancial relationships to disclose.

K.L.G. receives a stipend and tuition remission through the Knox Fellowship at Purdue University. She has no relevant nonfinancial relationships to disclose.

B.B. receives a salary from Purdue University and is supported by NIH NIDCD grant R01
DC00559. She has no relevant nonfinancial relationships to disclose.

C.W. receives a salary from Purdue University and support from NIH NIDCD grants R01 DC00559 and R01 DC014708. She has no relevant nonfinancial relationships to disclose. A.S. receives a salary from Purdue University and support from NIH NIDCD grant R01 DC00559. She has no relevant nonfinancial relationships to disclose.

\section{REFERENCES}

1. Smith A, Weber C. How stuttering develops: the multifactorial dynamic pathways theory. J Speech Lang Hear Res 2017;60(09):2483-2505

2. Yairi E, Ambrose NG. Early childhood stuttering I: persistency and recovery rates. J Speech Lang Hear Res 1999;42(05):1097-1112

3. Yairi E, Ambrose NG. Early Childhood Stuttering for Clinicians by Clinicians. Austin, TX: Pro-Ed; 2005

4. Ambrose NG, Yairi E. Normative disfluency data for early childhood stuttering. J Speech Lang Hear Res 1999;42(04):895-909

5. Gillam R, Logan K, Pearson N. Test of Childhood Stuttering. Austin, TX: Pro-Ed; 2009

6. Pantelopoulos A, Bourbakis N. A survey on wearable biosensor systems for health monitoring. Conf Proc IEEE Eng Med Biol Soc 2008; 2008:4887-4890

7. Luck S. An Introduction to the Event-Related Potential Technique. Cambridge, MA: MIT Press; 2014

8. Kreidler K, Hampton Wray A, Usler E, Weber C. Neural indices of semantic processing in early childhood distinguish eventual stuttering persistence and recovery. J Speech Lang Hear Res 2017; 60(11):3118-3134

9. Usler E, Weber-Fox C. Neurodevelopment for syntactic processing distinguishes childhood stuttering recovery versus persistence. J Neurodev Disord 2015;7(01):4

10. Mohan R, Weber C. Neural systems mediating processing of sound units of language distinguish recovery versus persistence in stuttering. J Neurodev Disord 2015;7(01):28

11. Walsh B, Tian F, Tourville JA, Yücel MA, Kuczek T, Bostian AJ. Hemodynamics of speech production: an fNIRS investigation of children who stutter. Sci Rep 2017;7(01):4034

12. Beal DS, Lerch JP, Cameron B, Henderson R, Gracco VL, De Nil LF. The trajectory of gray matter development in Broca's area is abnormal in people who stutter. Front Hum Neurosci 2015;9:89 
13. Chang SE, Zhu DC, Choo AL, Angstadt M. White matter neuroanatomical differences in young children who stutter. Brain 2015;138(Pt 3):694-711

14. Hosseini R, Walsh B, Tian F, Wang S. An fNIRSbased feature learning and classification framework to distinguish hemodynamic patterns in children who stutter. IEEE T Neur Sys Reh 2018;1:1

15. Alm PA. Stuttering in relation to anxiety, temperament, and personality: review and analysis with focus on causality. J Fluency Disord 2014;40:5-21

16. Kefalianos E, Onslow M, Block S, Menzies R, Reilly S. Early stuttering, temperament and anxiety: two hypotheses. J Fluency Disord 2012; 37(03):151-163

17. Gross JJ, John OP. Individual differences in two emotion regulation processes: implications for affect, relationships, and well-being. J Pers Soc Psychol 2003;85(02):348-362

18. Putnam SP, Rothbart MK. Development of short and very short forms of the Children's Behavior Questionnaire. J Pers Assess 2006;87(01):102-112

19. Vanryckeghem M, Brutten GJ. KiddyCAT Communication Attitude Test. San Diego, CA: Plural Publishing; 2006

20. Arnold HS, Conture EG, Key APF, Walden T. Emotional reactivity, regulation and childhood stuttering: a behavioral and electrophysiological study. J Commun Disord 2011;44(03):276-293

21. Jones R, Choi D, Conture E, Walden T. Temperament, emotion, and childhood stuttering. Semin Speech Lang 2014;35(02):114-131

22. Walsh B, Smith A. Physiological and behavioral indices of emotional processes in preschoolers who stutter. Presentation at: The Oxford Dysfluency Conference; September 2017; Oxford, England

23. Zengin-Bolatkale H, Conture EG, Walden TA. Sympathetic arousal of young children who stutter during a stressful picture naming task. J Fluency Disord 2015;46:24-40

24. Zengin-Bolatkale H, Conture EG, Walden TA, Jones RM. Sympathetic arousal as a marker of chronicity in childhood stuttering. Dev Neuropsychol 2018;43(02):135-151

25. Walsh B, Usler E. Autonomic correlates of speech production in preschoolers who stutter. Poster presentation at: The One World, Many Voices: Science and Community Joint World Congress; July 2018; Tokyo, Japan

26. Boey RA, Van de Heyning PH, Wuyts FL, Heylen L, Stoop R, De Bodt MS. Awareness and reactions of young stuttering children aged 2-7 years old towards their speech disfluency. J Commun Disord 2009;42(05):334-346

27. Craig A, Tran Y. Trait and social anxiety in adults with chronic stuttering: conclusions following meta-analysis. J Fluency Disord 2014;40:35-43

28. Usler E, Weber C. Neural correlates of emotional activity associated with childhood stuttering.
Poster presentation at: the American SpeechLanguage-Hearing Conference; November 2016; Philadelphia, PA

29. Karrass J, Walden TA, Conture EG, et al. Relation of emotional reactivity and regulation to childhood stuttering. J Commun Disord 2006;39(06): 402-423

30. Ntourou K, Conture EG, Walden TA. Emotional reactivity and regulation in preschool-age children who stutter. J Fluency Disord 2013;38(03): 260-274

31. Zimmermann G. Articulatory dynamics of fluent utterances of stutterers and nonstutterers. J Speech Hear Res 1980;23(01):95-107

32. MacPherson MK, Smith A. Influences of sentence length and syntactic complexity on the speech motor control of children who stutter. J Speech Lang Hear Res 2013;56(01):89-102

33. Smith A, Goffman L, Sasisekaran J, Weber-Fox C. Language and motor abilities of preschool children who stutter: evidence from behavioral and kinematic indices of nonword repetition performance. J Fluency Disord 2012;37(04):344-358

34. Walsh B, Mettel KM, Smith A. Speech motor planning and execution deficits in early childhood stuttering. J Neurodev Disord 2015;7(01):27

35. Usler E, Smith A, Weber C. A lag in speech motor coordination during sentence production is associated with stuttering persistence in young children. J Speech Lang Hear Res 2017;60(01):51-61

36. Spencer C, Weber-Fox C. Preschool speech articulation and nonword repetition abilities may help predict eventual recovery or persistence of stuttering. J Fluency Disord 2014;41:32-46

37. Carrow-Woolfolk E. Test for Auditory Comprehension of Language. 3rd ed. Circle Pines, MN: AGS; 1999

38. Dawson J, Stout C, Eyer J. Structured Photographic Expressive Language Test. 3rd ed. DeKalb, IL: Janelle Publications; 2003

39. Bankson N, Bernthal J. Bankson-Bernthal Test of Phonology. Austin, TX: Pro-Ed; 1990

40. Dollaghan C, Campbell TF. Nonword repetition and child language impairment. J Speech Lang Hear Res 1998;41(05):1136-1146

41. Gardner M. Test of Auditory-Perceptual Skills. San Francisco, CA: Children's Hospital of San Francisco; 1985

42. Bloodstein O, Ratner NB. A Handbook on Stuttering. 6th ed. Clifton Park, NY: Cengage Learning; 2008

43. Yairi E, Ambrose N. A longitudinal study of stuttering in children: a preliminary report. J Speech Hear Res 1992;35(04):755-760

44. Bostian AJ, Brown B, Weber C. Disfluency characteristics of children aged 4- to 5-years associated with persistence and recovery of stuttering. Poster presented at: The American Speech, Language, and 
Hearing Convention; November 2016; Philadelphia, PA

45. Miller J, Iglesias A. Systematic Analysis of Language Transcripts (SALT). Madison, WI: University of Wisconsin-Madison; 2006

46. Smith A, Zelaznik HN. Development of functional synergies for speech motor coordination in childhood and adolescence. Dev Psychobiol 2004;45 (01):22-33

47. Kraft SJ, Yairi E. Genetic bases of stuttering: the state of the art, 2011. Folia Phoniatr Logop 2012; 64(01):34-47
48. Erdemir A, Walden TA, Jefferson CM, Choi D, Jones RM. The effect of emotion on articulation rate in persistence and recovery of childhood stuttering. J Fluency Disord 2018;56:1-17

49. Yairi E, Ambrose NG, Niermann R. The early months of stuttering: a developmental study. J Speech Hear Res 1993;36(03):521-528

50. Throneburg RN, Yairi E. Durational, proportionate, and absolute frequency characteristic of disfluencies: a longitudinal study regarding persistence and recovery. J Speech Lang Hear Res 2001;44(01): $38-51$ 